\title{
STUDY OF PREVALENCE AND RESPONSE TO NEEDLE STICK INJURIES AMONG HEALTH CARE WORKERS IN A TERTIARY CARE HOSPITAL IN HYDERABAD, INDIA
}

\author{
B. Baburao' ${ }^{1}$ J Syam Sundar².
}

1. Assistant Professor, Department of Community Medicine, Osmania Medical College.

2. Assistant Professor, Department of Community Medicine, Government Medical College. Nizamabad.

\section{CORRESPONDING AUTHOR}

Dr B. Baburao,

Assistant Professor,

Department of Community Medicine,

Osmania Medical College,

Koti, Hyderabad.

Email Id: baburao1234@yahoo.com

\section{HOW TO CITE THIS ARTICLE:}

B. Baburao. J. Syam Sundar. "Study of Prevalence \& Reponse to Needle Stick injuries among health Care Workers in a Tertiary Care Hospital in Hyderabad. India." Journal of Evolution of Medical and Dental Sciences 2013; Vol2, Issue 23, June 10; Page: 4199-4204.

ABSTRACT:- BACKGROUND: Because of the environment in which they work, many health care workers are at risk of accidental needle stick injuries (NSI). OBJECTIVES: To study prevalence and response to needle stick injuries among health care workers. METERIALS AND METHODS:STUDY DESIGN: Cross-sectional study. SETTING: A tertiary care hospital in Hyderabad. PARTICIPENTS: 400, senior residents, junior residents, interns, undergraduate medical students, staff and student nurses and staff and student laboratory technicians. STATISTICAL ANALYSIS: proportions and chi-square test. RESULTS: The commonest clinical activity to cause the NSI was blood withdrawal (55\%), followed by suturing $(20.3 \%)$ and vaccination $(11.7 \%)$. The practice of recapping needles after use was still prevalent among HCWs (66.3\%). Some HCWs also revealed that they bent the needles before discarding (11.4\%). It was alarming to note that only 40 per cent of the HCWs knew about the availability of PEP services in the hospital. CONCLUSIONS: The occurrence of NSI was found to be quite common. Avoidable practices like recapping of needles were contributing to the injuries. Prevention of NSI is an integral part of prevention programs in the work place, and training of HCWs regarding safety practices indispensably needs to be an ongoing activity at a hospital.

KEYWORDS: Needle stick injury, health care workers

INTRODUCTION: Needle stick injuries (NSI) are wounds caused by sharps such as hypodermic needles, blood collection needles, iv annuals or needles used to connect parts of iv delivery systems. The causes include various factors like type and design of needle, recapping activity, handling/transferring specimens, collision between HCWs or sharps, during clean-up, manipulating needles in patient line related work, passing/handling devices or failure to dispose of the needle in 
puncture proof containers ${ }^{1}$. Because of the environment in which they work, many HCWs from physicians, surgeons, and nurses to housekeeping personnel, laboratory technicians and waste handlers are at an increased risk of accidental needle stick and sharps injuries. As a result, these workers are prone to occupational acquisition of various blood borne pathogens, including the microorganisms causing HIV/AIDS, hepatitis B and C, malaria, infectious mononucleosis, diphtheria, herpes, tuberculosis, brucellosis, spotted fever and syphilis ${ }^{1}$.According to a WHO study, the annual estimated proportions of health-care workers (HCW) exposed to blood-borne pathogens globally were $2.6 \%$ for $\mathrm{HCV}, 5.9 \%$ for $\mathrm{HBV}$, and $0.5 \%$ for $\mathrm{HIV}$, corresponding to about $16,000 \mathrm{HCV}$ infections and 66,000 HBV infections in HCW worldwide².

Because needle stick injuries are often under reported, health care institutions should not interpret low reporting rate as low injury rate. Injuries recorded through standard occupational reporting systems may underestimate the true injury rate, as much as 10 -fold ${ }^{3}$. Needle stick injuries have significant indirect consequences in health care delivery especially so in the developing countries, where already the qualified work force is limited with respect to the disease burden in the population. These injuries not only potentiate health consequences but also cause emotional distress in health care workers which results in missed workdays and directly affects the health care services and resources.

The present study addresses this important issue of NSI and aims at determining their occurrence among the health care workers in a tertiary care hospital in Hyderabad, the various factors responsible for needle stick injuries, the circumstances under which they occur and explores the responses of the health care workers after an injury.

MATERIAL AND METHODS: This study was carried out among the HCWs (both males and females) of Osmania Medical College and Osmania General Hospital, a tertiary care, teaching hospital in Hyderabad, India. The study group consisted of various HCWs including senior residents, junior residents, interns, undergraduate medical students, staff and student nurses and staff and student laboratory technicians. The study was carried out over two months from June to August of 2009 with participation from 400 HCWs.

Subjects were fully informed about the design and purpose of the study and a written informed consent was obtained. The study was carried out with the help of an anonymous, selfreporting questionnaire structured specifically to obtain both qualitative and quantitative data to identify predictive factors associated with NSIs.

Case definition of NSI in the present study included injuries caused by sharps such as hypodermic needles, blood collection needles, IV cannulas, suture needles, winged needle iv sets and needles used to connect parts of the iv delivery systems.

The HCWs who gave a history of NSI were directed to seek advice on PEP and infection control measures from the Regional STD Centre, Osmania General Hospital which has a NSI protocol in place.

Clearance of study protocol was obtained from the institutional ethics committee before the start of the study. Findings were analyzed under different headings to uncover various aspects of NSI. The statistical tools employed were-ratio, proportion pie charts, histograms and other basic methods of data interpretation 
FINDINGS: A total of 400HCWs participated in the study, including senior residents 60 (15\%), junior residents-30 (7.5\%), interns-70 (17.5\%), undergraduate medical students-70 (17.5\%), staff and student nurses $-40(10 \%)$ and $60(15 \%)$ respectively, staff and student laboratory technicians $70(17.5 \%)$.

Among the HCWs with NSIs, nurses had the highest percentage 36(90\%), followed by nursing students $52(86.6 \%)$, laboratory technicians $59(84.2 \%)$, interns $56(80 \%)$ junior residents $20(66.6 \%)$, senior residents $36(60 \%)$ and undergraduate students $33(47 . \%)$.

These are shown below table I

TABLE-I

\begin{tabular}{|l|l|l|}
\hline Health care workers & $\begin{array}{l}\text { Total of } \\
\text { participants } \\
\text { N=400 }\end{array}$ & $\begin{array}{l}\text { Having Needle stick } \\
\text { injury }\end{array}$ \\
\hline Senior Residents & $60(15 \%)$ & $36(60 \%)$ \\
\hline Junior Residents- & $30(7.5 \%)$ & $20(66.6 \%)$ \\
\hline Interns & $70(17.5 \%)$ & $56(80 \%)$ \\
\hline Undergraduate Medical Students & $70(17.5 \%)$ & $33(47 \%)$ \\
\hline Staff Nurses & $40(10 \%)$ & $36(90 \%)$ \\
\hline Student Nurses & $60(15 \%)$ & $52(86.6 \%)$ \\
\hline $\begin{array}{l}\text { staff and student laboratory } \\
\text { technicians }\end{array}$ & $70(17.5 \%)$ & $59(84.2 \%)$, \\
\hline
\end{tabular}

Seventy four per cent of HCWs were wearing gloves at the time of NSI, which included senior residents $(83.3 \%)$, interns $(85 \%)$, junior residents $(62 \%)$, undergraduates $(59 \%)$, staff nurses $(71 \%)$, student nurses $(60 \%)$ and laboratory technicians $(85 \%)$.

The length of the needle in most cases of NSI was medium sized (66.3\%), while large $(13.7 \%)$ and small sized needles (20\%) were also implicated. Seventy one per cent of the needles involved were hollow bored, with only 29 per cent being non-bored or curved.

The commonest clinical activity to cause the NSI was blood withdrawal (55\%), followed by suturing (20.3\%) and vaccination (11.7\%). About 13 per cent of the HCWs received the NSI due to patient aggressiveness. Recapping needles was a common cause of NSI (66.3\%)

After a NSI, majority of HCWs took action instantly (60\%), while 14 per cent took action later on the same day and 26 per cent did not take any action.

The action taken included washing the site with soap and water and/or cleaning the site with appropriate agents like alcohol, dettol or other antiseptic agents, reporting the incident to senior staff and seeking advice on NSI protocol (testing for HIV/HBV/HCV and PEP) from Regional STD Centre. It was noticed that 54 per cent of NSI occurred in second $1 / 3 \mathrm{rd}$ of the duty period, while 25 per cent occurred in the first $1 / 3 \mathrm{rd}$ and 21 per cent in the third $1 / 3 \mathrm{rd}$ of duty period. A large number of HCWs suffered stress after NSI (65\%).

The effect of NSI on work efficiency reveals that 48 per cent became more cautious, 48 per cent took better precautions, 1 per cent avoided such procedures, while in 2 per cent the NSI had no effect and 1 per cent of HCWs became more casual. Most of the HCWs were aware of the possibility 
that NSI could lead to the acquisition of diseases like HIV, hepatitis B and C, but not any other diseases. About 34 per cent said that they had read articles about NSI and thus knew how to avoid them (mainly senior and junior residents).

Sixty two per cent of HCWs confirmed that they would wash the area if needle contaminated with HIV seropositive blood came in contact with skin, whereas 22 per cent said that they would take PEP. When questioned about the steps to be taken after NSI, 14 per cent opted for PEP, while 34 per cent felt that a shot of tetanus toxoid was sufficient, 5 per cent felt that taking analgesics was enough and 45.5 per cent preferred to wash the area with soap and water. A small percentage of HCWs (1.5\%) said that they would tie the part with some material. When questioned about the response to bleeding at the site of NSI, 66 per cent said that they had washed the area with soap and water, while 47 per cent applied spirit/alcohol, 19 per cent used band aid, 0.5 per cent said that they expressed the blood from the spot, 21.4 per cent scratched the NSI spot, 4 per cent applied pressure to stop bleeding and 2 per cent left it as it was.

The practice of recapping needles after use was still prevalent among HCWs (66.3\%), with 59 per cent using both hands. Some HCWs also revealed that they bent the needles before discarding (11.4\%).

DISCUSSION: The present study addressed certain aspects of NSI in a busy tertiary care government hospital and derived some equivocal and some contrasting results. It was found that 73 per cent of HCWs had experienced NSI at some point in their careers. Among the HCWs, nurses were most prone to NSI, with 90 per cent of them having experienced it in the preceding one year.. This may be attributed to patient overload and different work culture in the Indian scenario. Several other studies had also shown high occurrence of NSI among nurses ${ }^{4-7}$. Apart from nurses the NSIs were more among nursing students, interns and resident doctors. Cervini and Bell ${ }^{8}$, have shown that post-exposure practices for NSI are inadequate among medical students and our findings corroborate this fact. Another study showed that increasing surgical experience lowered NSI rates16, and that specific training and passive prevention solutions seem more important than enhanced training and reporting guidelines in preventing NSIs.

Among the HCWs studied, 74 per cent were using gloves at the time of NSI, a figure which fell short of the figures shown by Askarian et al (96.2\%) 9 in Iran. Majority of the needles associated with NSI were of medium size, as this was the size most commonly used in patient care.

Certain clinical practices such as recapping needles were related more to the likelihood of being injured.

More than 50 per cent NSI cases, the HCW was involved in withdrawal of blood, as it is the most common activity involving manipulation of needle in patient. In the EPInet study10, 38 per cent NSI occurred during needle use, while 42 per cent occurred after use of needle and before its disposal. The comparative figures in the present study were 34 and 60 per cent, respectively.

About 65 per cent of HCWs suffered stress as a result of the NSI, by their own admission. Other studies showed similar statistics of stress (55\% in EPI net study) ${ }^{10}$.

Less than 50 per cent HCWs knew about the availability of PEP services in the hospital. This was higher than the figures in a study by Chacko and Isaac (31.6\%) ${ }^{11}$.

Almost all HCWs were aware that AIDS and hepatitis B and C can spread by NSI, but very few were aware of the large number of other diseases linked to NSI. Thus, increasing staff 
awareness and educating them on NSI issues is a felt need, as only 34 per cent read textbooks or articles about NSI and only 29 per cent were aware of the average risk of HIV/AIDS from NSI as against 87.8 per cent in a study from Iran ${ }^{12}$.

Most HCWs in the present study were of the opinion that education, training, better safety devices, decreased patient load per HCW, positive work environment and following standard precautions can help prevent NSI.

According to a CDC report, use of safety engineered devices would reduce NSIs by 76 per cent $^{13}$. There is much room for improvement in protecting the HCWs from NSI, which can be accomplished through a combination of comprehensive programmes, including stress on institutional behaviour and device related factors that contribute to the occurrence of these injuries, seeking alternatives to use of needles wherever possible, using newer devices with safety features, ensuring adequate training in safe use and disposal of needles, putting in place a culture of accident reporting, especially sharps-related, and following preventive practices like vaccinations for hepatitis B, as also stressed by several others ${ }^{13-15}$.

Some institutions in India ${ }^{16}$, have a staff student health service facility in place, which maintains records, and registers the incidence of NSI and has protocols for management and followup of NSI cases. This is a dire necessity in all large health care facilities with a large turnover of patients and a high rate of NSI.

In conclusion, NSIs were observed in all categories of HCWs. There is a scope for improvement in safety protocols. Preventive strategies have to be devised and reporting of NSI need to be made mandatory. Issues requiring attention include use of safety engineered devices (SED), recording and reporting of incidents, training of all HCWs in handling and disposal of sharps, establishing a staff student health service and inculcating a responsible attitude among HCWs.

\section{REFERENCE:}

1. Wilburn SQ. Needle sticks and sharps injury prevention. Online J Issues Nursing 2004; 9: Manuscript 4.

2. Pruss-Ustun A, Rapiti E, Hutin Y. Sharps injuries: Global burden of disease from sharps injuries to health-care workers. Geneva: World Health Organization; 2003. (WHO Environmental Burden of Disease Series, No. 3)

3. Elder A, Paterson C. Sharps injuries in UK health care: A review of injury rates, viral transmission and potential efficacy of safety devices. Occup Med (Lond) 2006;56:566-74.

4. Nili T, Amal MS, Shaul SS. The health beliefs of hospital staff and the reporting of needlestick injury. J Clin Nurs 2006; 15: 1228-39.

5. Nagao Y, Baba H, Torii K, Nagao M, Hatakeyama K, Linuma Y, et al. A long term study of sharps injuries among health care workers of Japan. Am J Infect Control 2007; 35: 407-11.

6. Smith DR, Leggat PA. Needlesticks and sharps injuries among nursing students. J Adv Nurs 2005; $51:$ 449-55.

7. Alamgir H, Cvitkovich Y, Astrakianakis G, Yu S, Yassi A. Needlestick and other potential blood and body fluid exposures among health care workers in British Columbia, Canada. Am Infect Control 2008; 36: 12-21.

8. Cervini P, Bell C. Brief report: Needlestick injuries and inadequate post exposure practice in medical students. J Gen Intern Med 2005; 20: 419-21. 
9. Askarian M, Shaghaghian S, McLaws ML. Needlestick injuries among nurses of Fars Province, Iran. Ann Epidemiol 2007; 17: 988-92.

10. International Health Care Workers Safety Center. Exposure prevention information network (EPI net) Data reports; Charlottesville: University of Virginia; 1999.

11. Chacko J, Isaac R. Percutaneous injuries among medical interns and their knowledge and practice of post-exposure prophylaxis for HIV. Indian J Public Health 2007; 51: 127-9.

12. Askarian M, Malekmakan L. The prevalence of needlestick injuries in medical, dental, nursing and midwifery students at the university teaching hospitals of Shiraz, Iran. Indian J Med Sci 2006; 60: 227-32.

13. Lal P, Singh MM, Malhotra R, Ingle GK. Perception of risk and potential occupational exposure to HIV/AIDS among medical interns in Delhi. J Commun Dis 2007; 39: 95-9.

14. Clarke SP. Hospital work environments, nurse characteristics, and sharps injuries. Am J Infect Control 2007; 35: 302-9.

15. Zafar A, Aslam N, Nasir N, Meraj R, Mehraj V. Knowledge, attitudes and practices of healthcare workers regarding needlestick injuries at a tertiary care hospital in Pakistan. $J$ Pak Med Assoc 2008; 58: 57-60.

16. Jayanth ST, Kirupakaran H, Brahmadathan KN, Gnanaraj L, Kang G. Needlestick injuries in a tertiary care hospital. Indian J Med Microbiol 2009; 27: 44-7. 